DOI: $10.24850 /$ j-tyca-2018-03-07

Artículo

\title{
Estimación de la exposición a elevados contenidos de fluoruro en agua potable en distintas comunidades de Guanajuato, México
}

\section{Estimation of exposure to high fluoride contents in groundwater supply in some villages in Guanajuato, Mexico}

José Iván Morales-Arredondo† ${ }^{1}$

María Aurora Armienta²

Ramiro Rodríguez 3

${ }^{1+}$ Universidad Nacional Autónoma de México, Instituto de Geofísica, Cd. Universitaria, Ciudad de México, México, ivanma@geofisica.unam.mx

2Universidad Nacional Autónoma de México, Instituto de Geofísica, Cd. Universitaria, Ciudad de México, México, victoria@geofisica.unam.mx

3Universidad Nacional Autónoma de México, Instituto de Geofísica, Cd. Universitaria, Ciudad de México, México, ramiro@geofisica.unam.mx

Autor de correspondencia: José Iván Morales-Arredondo†

\section{Resumen}

Se llevó a cabo un estudio para estimar los niveles de riesgo por exposición para desarrollar fluorosis dental y esquelética en distintas comunidades en Santa Cruz de Juventino Rosas (JR), estado de Guanajuato, México. El estudio incluyó el análisis químico de la concentración de fluoruros en muestras de agua de 23 pozos durante los años 2011 hasta 2016; se realizó el cálculo para estimar la exposición a fluoruros (EEF) y una encuesta epidemiológica (EEp) usando el Índice de 
Dean (ID). EL rango de concentración de fluoruros en los pozos fue de 0.15 a $3.24 \mathrm{mg} / \mathrm{l}$. Doce pozos muestreados exceden el límite permisible de calidad en relación con la concentración de fluoruros, establecido por la norma oficial mexicana NOM-127-SSA1-1994, que es de $1.5(\mathrm{mg} / \mathrm{l})$ en agua para consumo humano. Altas concentraciones de $\mathrm{F}^{-}$en el agua subterránea se han asociado con flujos geotérmicos ascendentes. De acuerdo con los resultados de EEF $(0.01-1.8 \mathrm{mg} / \mathrm{kg} / \mathrm{d})$, las personas de tres asentamientos rurales con rangos de edad entre 16 y 50 años están en riesgo potencial para generar fluorosis dental; los resultados de la EEp confirmaron esta situación. El $23 \%$ de la población encuestada mostró evidencias de fluorosis dental, la mayoría gente joven; más de $50 \%$ de los individuos con evidencias de fluorosis dental tienen entre 16 y 50 años. Se considera que un factor importante para el desarrollo de fluorosis dental es el continuo consumo de agua enriquecida en fluoruros. Algunas de las personas encuestadas viven en el sitio desde hace más de 20 años.

Palabras clave: fluorosis dental, México, patrones de consumo de agua.

\section{Abstract}

A study was carried out to estimate the level of fluoride exposure and the human health risk of developing dental and skeletical fluorosis in some of the villages in Santa Cruz de Juventino Rosas (JR), state of Guanajuato, Mexico. The study included a fluoride concentration analysis of groundwater samples from 23 wells during the years 2010 to 2016, estimation of exposure to fluoride (EEF), and an epidemiological survey (ES) using Dean's Index (DI). Fluoride concentrations ranged from 0.51 to $324 \mathrm{mg} / \mathrm{l}$. Twelve sampled wells exceeded the national guideline values for fluoride concentrations (NOM-127-SSA1-1994: $1.5 \mathrm{mg} / \mathrm{l}$ ). High $\mathrm{F}^{-}$concentrations are associated with ascending geothermal flow. According to EEF results $(0.01-1.8 \mathrm{mg} / \mathrm{kg} / \mathrm{d})$, people from three small towns, from 16 to 50 years old, were at potential risk of dental fluorosis. This was confirmed by the results from the ES, with $23 \%$ of the people surveyed showing evidence of dental fluorosis, mostly young individuals. More than $50 \%$ of the people with evidence of dental fluorosis were between 16 to 50 years old. The ongoing consumption of fluoride-rich water is considered to be an important factor for developing dental fluorosis, and some of the people surveyed have lived at the study site for about 20 years.

Keywords: Dental fluorosis, Mexico, water consumption patterns. 
Recibido: $25 / 03 / 2017$

Aceptado: 08/11/2017

\section{Introducción}

En distintas regiones del mundo se han reportado afectaciones a la salud debido a la ingesta de agua con elevados contenidos de $\mathrm{F}^{-}$(Griffin, Beltran, Lockwood, \& Barker, 2002; Soto-Rojas, Ureña-Cirret, \& MartinezMier, 2004; Armienta \& Segovia, 2008). Agua subterránea con altos contenidos de $\mathrm{F}^{-}$de origen natural por lo usual se asocia con agua geotérmica que interactúa con rocas ricas en $\mathrm{F}^{-}$, sobre todo rocas volcánicas (Ruiz, Kesler, \& Jones, 1985; Morales, Villanueva-Estrada, Rodríguez, \& Armienta, 2015). La disolución de fluorita, de silicatos contenidos en rocas ígneas y, en algunos casos, mármoles dolomíticos se han identificado como las principales fuentes de $\mathrm{F}^{-}$en agua subterránea (Petersen, Essene, \& Peacor, 1982; Ravishankar, 1987; Levy, Schramke, Esposito, Erickson, \& More, 1999). Parámetros como pH, temperatura, profundidad de pozos, hidrogeología y características tectónicas son factores involucrados en la presencia de fluoruros (Rodríguez et al., 2001; Armienta \& Segovia 2008; Morales et al., 2015). El agua subterránea con altos contenidos de $\mathrm{F}^{-}$, que es usada para abastecer poblaciones urbanas, representa un riesgo potencial por exposición (Rivett et al., 2006; EPA, 2011). La ingesta de agua con elevados contenidos de $\mathrm{F}^{-}$durante periodos largos de tiempo puede producir acumulación de fluoruro en tejidos calcificados y ligamentos, deformación de huesos, desorden renal y fluorosis dental y esquelética (McDonagh et al., 2000; EPA, 2011; Griffin et al., 2002). En México se ha reportado fluorosis dental debida a una elevada concentración de fluoruros en agua subterránea que es usada para consumo humano en ciudades como Aguascalientes, Chihuahua, Durango, Hermosillo, Salamanca, San Miguel de Allende y San Luis Potosí (Grimaldo et al., 1995; Díaz-Barriga et al., 1997; Mendoza, 1999; Alarcón-Herrera, Martín-Domínguez, Trejo-Vázquez, \& Rodríguez-Dozal, 2001; Grijalva-Haro, Barba-Leyva, \& Laborín-Alvarez, 2001; TrejoVázquez \& Bonilla-Petriciolet, 2001; Hurtado-Jiménez \& Gardea-Torredey, 2005; Betancourt-Lineares, Irigoyen-Camacho, Mejía-González, ZepedaZepeda, \& Sánchez-Pérez, 2013; SAPASMA, 2006). Santa Cruz de 
Juventino Rosas (JR) es una pequeña comunidad localizada en el bajío guanajuatense, en el estado de Guanajuato, centro de México (Figuras 1 y 2), donde la agricultura y ganadería son las principales actividades económicas, y el agua subterránea es la principal fuente de abastecimiento del vital líquido (Rodríguez et al., 2001). Más de 2000 pozos componen la red de suministro de agua en el bajío guanajuatense; en JR existen 380 pozos agrícolas, urbanos e inactivos; 66 de ellos suministran agua potable. De estos últimos se muestrearon 23 pozos, 10 de ellos presentaron elevados contenidos de $\mathrm{F}^{-}$. En otras ciudades del estado de Guanajuato se han reportado afectaciones relacionadas con la elevada presencia de $\mathrm{F}^{-}$(Ortíz-Burgos, Vargas-Garcidueñas, \& OvalleCastro, 1996; Cervantes-González, Ortíz-Burgos, \& Wilbert-Ovalle, 1997). De acuerdo con Morales et al. (2015), el agua subterránea de JR presenta características geotérmicas con concentraciones elevadas de $\mathrm{F}^{-}$ de origen natural, tales concentraciones exceden el límite máximo permitido para concentración de fluoruros por la norma mexicana para agua de consumo humano (NOM-127-SSA1-1994; $\mathrm{F}=1.5 \mathrm{mg} / \mathrm{l}$ ). Rodríguez, Morales-Arredondo y Rodríguez (2016) calcularon los valores de fondo, los cuales presentaron una concentración de $1.93 \pm 0.75 \mathrm{mg} / \mathrm{l}$. El objetivo planteado en el presente estudio es estimar la exposición a fluoruros en el agua de consumo y evaluar el riesgo de la población de Santa Cruz de Juventino Rosas, Guanajuato, México, de desarrollar fluorosis debido a la misma; para ello se analizó el contenido de F- en el agua subterránea.

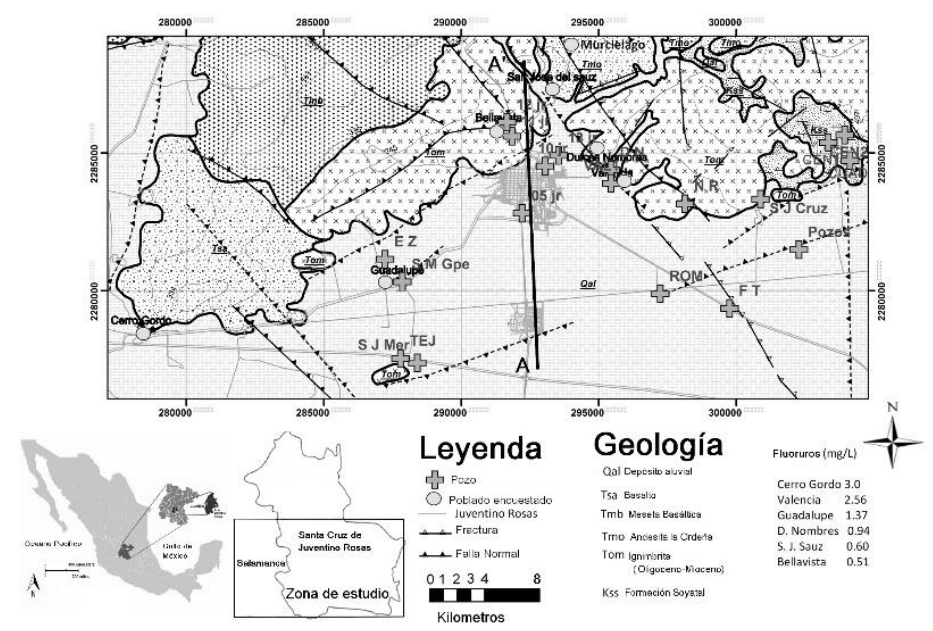

Figura 1. Geología y localización de los pozos muestreados en la comunidad rural, y en la zona encuestada en Juventino Rosas. La línea $A A^{\prime}$ simboliza la zona donde se obtuvo un perfil estratigráfico. 


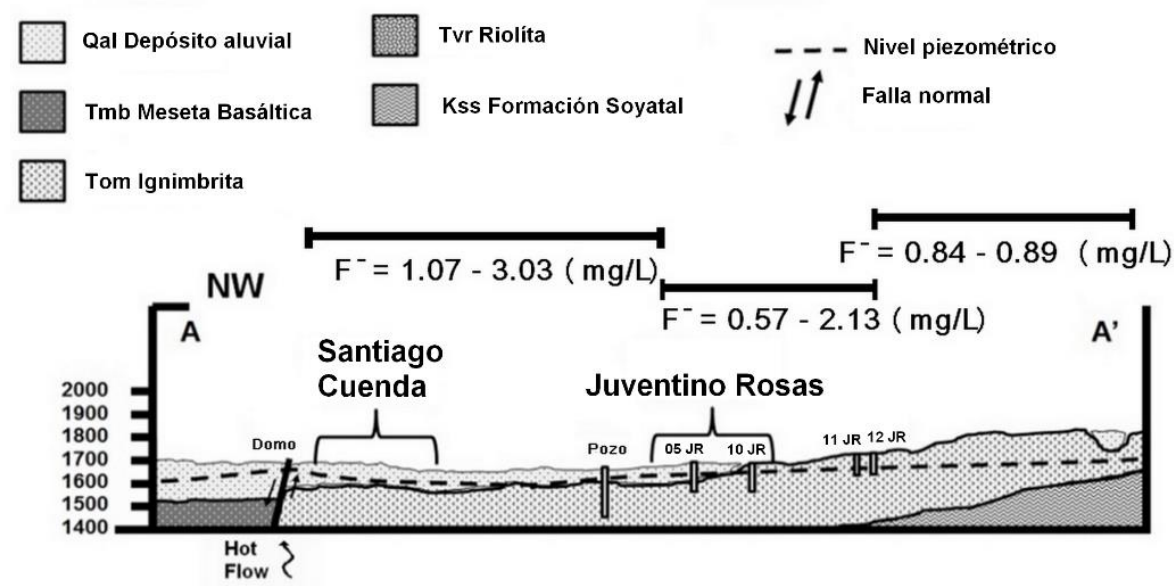

Figura 2. Perfil estratigráfico $A A^{\prime}$ de la zona de estudio. En el perfil se observan algunos de los pozos muestreados y el rango de concentración de fluoruros en la zona de recarga $(0.84-0.89 \mathrm{mg} / \mathrm{l})$, la zona de piedemonte $(0.57-2.13 \mathrm{mg} / \mathrm{l})$ y la planicie aluvial $(1.07-3.03 \mathrm{mg} / \mathrm{l})$.

\section{Área de estudio}

\section{Localización, geología e hidrogeología}

El municipio de Juventino Rosas está localizado en la Faja Volcánica TransMexicana. Hacia el norte, la Sierra de Guanajuato limita el valle (Figura 1). En el bajío guanajuatense predomina un clima semiárido, con una precipitación promedio de 600 a $700 \mathrm{~mm}$ por año. El basamento geológico está definido por rocas volcanosedimentarias del Cretácico y sedimentos calcáreos arcillosos metamorfizados de la Formación Soyatal (CercaMartínez, Aguirre-Díaz, \& López-Martínez, 2000). Suprayaciendo el basamento, hay rocas riolíticas y basaltos (Quintero, 1999); estas unidades de 100 a $150 \mathrm{~m}$ de espesor alojan el acuífero principal y el más explotado (CEAG, 2000) (Figuras 1 y 2). Dos eventos tectónicos afectaron gran parte de la zona: el primero está relacionado con la orogenia 
laramide, mientras que el segundo es producto de una fase extensional con intenso magmatismo. Ambos eventos produjeron fallas y fracturas en la zona, con una dirección preferencial NE-SO, E-O y NO-SE (CercaMartínez et al., 2000). Estas fallas permiten el ascenso de flujos geotérmicos que pueden contener elementos como $\mathrm{F}^{-} \mathrm{y}$ otros reportados, como As y ${ }^{222} \mathrm{Rn}$ (Morales et al., 2015; Rodríguez, Morales, Armienta, Villanueva, \& Segovia, 2015) (Figuras 1, 2 y 3). La cuenca sedimentaria está rellena de sedimentos derivados de la meteorización de rocas volcánicas que rodean el sitio. Además, hay sedimentos lacustres de granulometría variable (Orozco-Esquivel, Nieto-Samaniego, \& AlanizAlvarez, 2002). La cuenca sedimentaria aloja el acuífero más somero con características semiconfinantes, que está a una profundidad de 20 a 25 $\mathrm{m}$ y cuya explotación ha provocado procesos de subsidencia.

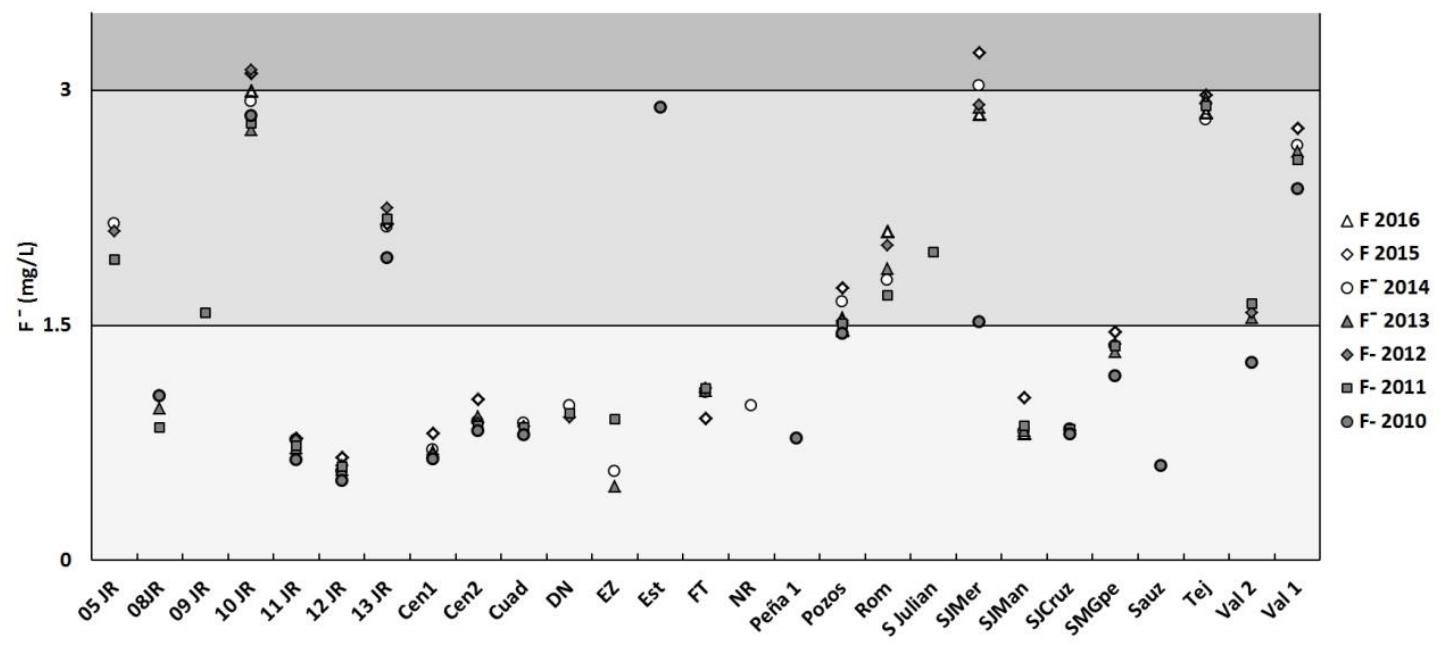

Figura 3. Concentración de fluoruros en pozos muestreados desde 2010 hasta 2016.

\section{Metodología}

Se llevaron a cabo campañas de muestreo y monitoreo en pozos urbanos, rurales y agrícolas de Juventino Rosas, del año 2010 al 2016. Estas 
campañas se realizaron una o dos veces al año, durante los meses de julio y octubre; las muestras de agua se recolectaron como describe la normatividad mexicana (NOM-230-SSA1-2002). Los 23 pozos monitoreados están localizados en tres zonas (Figura 1): al noroeste (Dulces Nombres = DN; Juventino Rosas = JR; 13JR; 12JR; 11JR; 09JR; 05JR; Emiliano Zapata = EZ); al noreste (Centeno 1 = Cen1; Centeno 2 = Cen2; Cuadrilla = Cuad; Nuevo Rocillo = NR; San Juan Cruz = SJCruz; San José Manantial = SJMan), y al sur (Valencia $1=$ Val1; Valencia $2=$ Val2; 10JR; 08JR; Franco Tavera = FT; Pozos = Pozos; Romerillo = Rom; San José Merino = SJMer; Santa María Guadalupe = SMGpe; Tejera = Tej). El análisis de la concentración de fluoruros se realizó en el Laboratorio de Química Analítica, en el Instituto de Geofísica de la Universidad Nacional Autónoma de México (UNAM), siguiendo el método analítico APHA-AWWA-WWF (2005). Las concentraciones de $\mathrm{F}^{-}$fueron medidas con un potenciómetro con electrodo selectivo Thermo Scientific Orion 5 Star. Se añadió solución TISAB a los estándares y a las muestras, para ajustar la fuerza iónica y evitar la formación de complejos.

La estimación de la exposición a fluoruros (EEF) (ecuación (1)) se evaluó en seis comunidades rurales (Tabla 1, Figura 1, Figura 2). Para realizar los cálculos, se tomó en cuenta que en toda la zona la única fuente de suministro de agua potable y de $\mathrm{F}^{-}$es la que se extrae a través de pozos profundos y que es usada para todas las actividades por la población. Se tomaron en cuenta valores estándar para la masa corporal de la población y para consumo de agua por rangos de edad (Environment Canada, 1993; Ortíz, Castro, Turrubiates, Milan, \& Díaz-Barriga, 1998). Para los cálculos se utilizó la siguiente ecuación:

$\mathrm{EEF}=([\mathrm{F}-] * \mathrm{CH} 20) /$ Wind $(1)$

Donde:

EEF = estimación de exposición a fluoruros $(\mathrm{mg} / \mathrm{kg} /$ día $)$.

$\left[\mathrm{F}^{-}\right]=$concentración de fluoruros en agua $(\mathrm{mg} / \mathrm{l})$.

$\mathrm{CH} 2 \mathrm{O}=$ consumo de agua por edad ( $/$ día $)$.

Wind $=$ masa corporal por edad por individuo $(\mathrm{kg})$. 
Tabla 1. Población encuestada en Juventino Rosas y sus comunidades agrícolas.

\begin{tabular}{|l|c|c|}
\hline Comunidad rural & Total de habitantes & Individuos encuestados \\
\hline Bellavista & 104 & 14 \\
\hline San José del Sauz & 483 & 26 \\
\hline Murciélago & 731 & 38 \\
\hline Dulces Nombres & 1258 & 19 \\
\hline Valencia & 337 & 39 \\
\hline Guadalupe & 391 & 11 \\
\hline Cerro Gordo & 1591 & 170 \\
\hline Total & $\mathbf{4 8 9 5}$ & $\mathbf{3 1 7}$ \\
\hline
\end{tabular}

Además, se realizó una encuesta epidemiológica y de patrones de consumo en la población en julio de 2013. En dicha encuesta se consideraron a) patrones de consumo de agua, b) evidencias visuales de afectaciones a la salud relacionadas con el consumo de elevadas concentraciones de $\mathrm{F}^{-}$a través de la ingesta de agua con el índice de Dean (ID) (Dean, 1934). Se utilizó una escala para diagnosticar e identificar los diferentes niveles de fluorosis dental (cuestionable, muy ligero, ligero, moderado y severo). Las evidencias cuestionables fueron descartadas del análisis. La encuesta fue llevada a cabo en seis comunidades (Tabla 1, Figura 1, Figura 2). La encuesta epidemiológica se hizo cara a cara en las comunidades y participaron diversas personas. Un grupo de especialistas en epidemiología de la Universidad de Guanajuato ayudó en la identificación de dichas afectaciones. Las personas encuestadas se clasificaron en estratos por familias. El total de la población era de $N=4$ 895. La encuesta epidemiológica consideró un método no probabilístico con rutas aleatorias y muestreo simple, donde todos los individuos de la población objetivo tenían la misma probabilidad de participar (Gómez, 1990; Rubio, 2009). La encuesta consideró edad, sexo, uso del agua y su manejo en el hogar, frecuencia de consumo de agua, percepción de agua con temperatura elevada; se evaluaron las posibles afectaciones a la salud relacionadas con la exposición a elevados contenidos de $\mathrm{F}^{-}$como fluorosis dental usando el ID. Además de agua potable existen otras rutas de exposición, como consumo de sal fluorurada, suplementos alimenticios 
y pasta de dientes, las cuales no se consideraron en este trabajo, aunque pudieran ser un factor a tomar en cuenta. Las personas encuestadas participaron de modo voluntario en el estudio, con consentimiento informado sin ninguna toma de muestra; su información es confidencial. En todo caso, las personas podían declinar a participar en el estudio y la información vertida aquí no tiene otro fin más que el de la investigación observacional, donde se estudian posibles factores asociados con la generación de fluorosis. El protocolo de investigación ha sido aprobado por un comité de bioética de la Universidad Nacional Autónoma de México.

El tamaño de la muestra se calculó de acuerdo con el siguiente criterio estadístico (Kish, 1982; Méndez, Namihira, Moreno, \& Sosa-de-Martinez, 2001):

$n^{\prime}=\left(z^{2} * p q\right) /(e \%)^{2}(2)$

Donde:

$n^{\prime}=$ tamaño de muestra estimada.

$z^{2}=$ es la abscisa de una curva normal que corta un área a en la distribución (1- a igual al nivel de confianza deseado de 95\%).

$p=$ proporción estimada de un atributo que está presente en la población; $p$ varía entre 0.4 y 0.6 .

$q=1-p$.

$e \%=$ el nivel deseado de precisión. Se acepta un error de $5 \%$. Por razones de ajuste, se eligió 5.33 (Gómez, 1990).

La ecuación (2) se puede ajustar para definir el tamaño de la muestra de la población a ser encuestada utilizando el tamaño de la población total que vive en las comunidades:

$n^{\prime \prime}=n^{\prime} /\left(1+\left(n^{\prime}-1\right) / N\right)(3)$

Donde: 
$n^{\prime \prime}=$ tamaño de la muestra ajustado a una población conocida.

$N=$ población total seleccionada de las comunidades: 4895 .

Reemplazando los valores propuestos:

$n^{\prime \prime}=[(1.96 * 1.96) *(0.5 * 0.5) /(0.0533 * 0.533)] /\{1+[((1.96 * 1.96) *(0.5 * 0.5) /$

$(0.0533 * 0.0533))-1] / 4895\}=316.284172$

Ello proporciona un tamaño de muestra adecuado y representativo de 316 individuos. Esta muestra tiene un nivel de confianza de $95 \%$ y un error máximo de $5.33 \%$ sobre una población total de 4895 habitantes. Las familias (casas) se seleccionaron siguiendo una secuencia de números aleatorios. El total de población encuestada comprende 317 habitantes: 157 hombres y 160 mujeres, divididos en grupos por edad; niños de 1 a 10 años (74 personas); adolescentes de 11 a 15 años (25 personas); gente joven de 16 a 25 años (65 personas); adultos de 26 a 50 años (111 personas), y personas de la tercera edad mayores de 50 años (40 personas).

A todos los participantes se les preguntó si su lugar de residencia había sido el mismo desde su nacimiento o si eran de otro sitio. En representación de los niños pequeños, sus tutores respondieron por ellos. Es necesario mencionar que en todas las comunidades donde se desarrolló el estudio, la gente labora en el campo, lejos de sus casas, y muchos de ellos sólo pasan el fin de semana con sus familias. En la mayoría de los casos, el padre de familia es el que trabaja todo el día, pero hay situaciones en las que ambos padres trabajan; esto provocó que en algunas comunidades no se encontraran personas para realizar las encuestas; asimismo, en algunas comunidades, como Dulces Nombres, la gente estaba renuente a participar en las encuestas, mientras que en Cerro Gordo la gente participó de forma activa; todas estas situaciones provocaron un desbalance en el tamaño de la muestra de individuos encuestados por comunidad.

\section{Resultados y discusión}


Los resultados presentan las concentraciones de fluoruros en pozos muestreados desde 2010 hasta 2016 (Figura 3). También incluyen datos indispensables para calcular la estimación de la exposición a fluoruros. Se reportan resultados de temperatura, conductividad eléctrica, sólidos disueltos totales, $\mathrm{pH}$ y dureza del agua. Los pozos con mayor temperatura y valores bajos de dureza presentan elevados contenidos de $\mathrm{F}^{-}$(Figura 3 ). Las muestras con un rango de $\mathrm{pH}$ de 6.61 a 8.04 y de CE entre 494 y 1 $100(\mu \mathrm{S} / \mathrm{cm})$ son las que tienen los valores más elevados de $\mathrm{F}^{*} ;$ no se observa una tendencia específica.

\section{Campaña de monitoreo}

Se efectuaron siete campañas de monitoreo de 2010 a 2016 (Figura 3, Tabla 2). En algunos pozos, las concentraciones de fluoruros fueron superiores al límite máximo permitido por la NOM-127-SSA1-1994. ( $F^{-}=$ $1.5 \mathrm{mg} / \mathrm{l}$ ) (Tabla 2).

Tabla 2. Concentración de fluoruros y parámetros físico-químicos de las muestras de agua colectadas.

\begin{tabular}{|c|c|c|c|c|c|c|c|c|c|c|c|c|}
\hline Pozos & $\begin{array}{l}\text { Temp } \\
{ }^{\circ} \mathrm{C} \\
\text { (prom } \\
\text { edio) }\end{array}$ & $\begin{array}{l}\text { CE } \\
(\mu S \\
/ \mathrm{cm} \\
)\end{array}$ & pH & $\begin{array}{l}\text { Dur } \\
\text { eza } \\
\text { (mg } \\
\text { /L) }\end{array}$ & $\begin{array}{l}\text { STD } \\
(\mathrm{mg} / \mathrm{I} \\
)^{2}\end{array}$ & $\begin{array}{l}\mathrm{F}^{-} \\
(\mathrm{mg} \\
/ \mathrm{l}) \\
201 \\
6\end{array}$ & $\begin{array}{l}F^{-} \\
(\mathrm{mg} / \mathrm{I} \\
) 2015\end{array}$ & $\begin{array}{l}F^{-} \\
(\mathrm{mg} / \mathrm{l} \\
) 2014\end{array}$ & $\begin{array}{l}\mathrm{F}^{-} \\
(\mathrm{mg} / \mathrm{I} \\
)^{2013}\end{array}$ & $\begin{array}{l}F^{-} \\
(\mathrm{mg} / \mathrm{I} \\
2012\end{array}$ & $\begin{array}{l}F^{-} \\
(\mathrm{mg} / \mathrm{I} \\
) 2011\end{array}$ & $\begin{array}{l}F^{-} \\
(\mathrm{mg} / \mathrm{l} \\
) 2010\end{array}$ \\
\hline $05 \mathrm{JR}$ & 34.0 & 751 & 7.27 & $\begin{array}{l}114 . \\
7\end{array}$ & 423.0 & & & 2.15 & & 2.1 & 1.92 & \\
\hline 08JR & 30.3 & 970 & 7.3 & & & & & & 0.972 & 1.05 & 0.85 & 1.05 \\
\hline $09 \mathrm{JR}$ & 29.5 & 610 & 7.13 & & & & & & & & 1.58 & \\
\hline $10 \mathrm{JR}$ & 38.0 & 590 & 6.82 & 47.0 & 358.2 & 3 & 3.11 & 2.93 & 2.75 & 3.13 & 2.79 & 2.84 \\
\hline $11 \mathrm{JR}$ & 27.3 & 629 & 6.86 & $\begin{array}{l}153 . \\
3\end{array}$ & 362.2 & & 0.78 & 0.768 & 0.715 & 0.77 & 0.73 & 0.64 \\
\hline
\end{tabular}




\begin{tabular}{|c|c|c|c|c|c|c|c|c|c|c|c|c|}
\hline $12 \mathrm{JR}$ & 26.1 & 660 & 6.7 & $\begin{array}{l}143 . \\
6\end{array}$ & 321.3 & & 0.66 & 0.569 & 0.58 & 0.603 & 0.6 & 0.51 \\
\hline $13 \mathrm{JR}$ & 32.4 & 594 & 6.61 & $\begin{array}{l}113 . \\
5\end{array}$ & 356.3 & & 2.15 & 2.13 & 2.18 & 2.25 & 2.18 & 1.93 \\
\hline Cen1 & 25.9 & 881 & 6.76 & & & & 0.81 & 0.706 & 0.694 & 0.672 & & 0.648 \\
\hline Cen2 & 27.7 & 955 & 6.84 & $\begin{array}{l}407 . \\
7\end{array}$ & & & 1.03 & 0.888 & 0.918 & 0.876 & 0.86 & 0.827 \\
\hline Cuad & 26.0 & $\begin{array}{l}861 . \\
5\end{array}$ & 6.73 & & & & & 0.874 & & 0.854 & 0.85 & 0.799 \\
\hline DN & 33.6 & 459 & 6.31 & $\begin{array}{l}102 . \\
8\end{array}$ & 282.4 & & & 0.984 & & 0.911 & 0.94 & \\
\hline $\mathrm{EZ}$ & 24.3 & 646 & 7.16 & $\begin{array}{l}118 . \\
5\end{array}$ & 371.3 & & & 0.569 & 0.471 & & 0.9 & \\
\hline Est & 50.0 & & & & & & & & & & & 2.89 \\
\hline FT & 37.5 & 595 & 7.32 & 74.8 & 348.9 & & 0.908 & 1.07 & 1.08 & 1.1 & 1.1 & \\
\hline NR & 34.0 & 473 & 6.42 & $\begin{array}{l}127 . \\
3\end{array}$ & 281.9 & & & 0.984 & & & & \\
\hline Peña 1 & 29.0 & & & & & & & & & & & 0.78 \\
\hline Pozos & 46.7 & 648 & 7.11 & 60.3 & 333.1 & 1.47 & 1.74 & 1.65 & 1.55 & 1.53 & 1.51 & 1.45 \\
\hline Rom & 39.9 & 577 & 8.04 & 14.0 & 357.2 & 2.1 & & 1.79 & 1.86 & 2.01 & 1.69 & \\
\hline $\begin{array}{l}\text { S } \\
\text { Julian }\end{array}$ & 40.9 & & & & & & & & & & 1.97 & \\
\hline SJMer & 48.4 & 494 & 7.52 & 36.3 & 363.3 & 2.85 & 3.24 & 3.03 & 2.89 & 2.91 & & 1.52 \\
\hline SJMan & 26.2 & $\begin{array}{l}781 . \\
5\end{array}$ & 7.15 & & & $\begin{array}{l}0.81 \\
1\end{array}$ & 1.04 & 0.821 & 0.828 & & 0.86 & \\
\hline SJCruz & 28.5 & 696 & 6.76 & $\begin{array}{l}246 . \\
5\end{array}$ & 335.1 & & & 0.838 & & 0.826 & 0.84 & 0.806 \\
\hline SMGpe & 29.2 & 576 & 7.36 & 49.0 & 369.5 & & 1.46 & 1.37 & 1.33 & 1.38 & 1.37 & 1.18 \\
\hline Sauz & 26.0 & & & & & & & & & & & 0.602 \\
\hline Tej & 46.3 & 517 & 7.32 & 40.1 & 334.8 & 2.86 & 2.97 & 2.81 & 2.95 & 2.92 & 2.9 & \\
\hline Val 2 & 29.9 & 1100 & 7.27 & & & & & & 1.55 & 1.58 & 1.64 & 1.26 \\
\hline Val 1 & 32.0 & 660 & 7.14 & 46.6 & 345.7 & & 2.76 & 2.65 & 2.61 & & 2.56 & 2.37 \\
\hline
\end{tabular}




\section{Estimación de la exposición a fluoruros (EEF)}

La concentración de fluoruros en agua subterránea en pozos muestreados de la comunidad rural fue usada para calcular el EEF. Se tomaron en cuenta valores estándar de la masa corporal de individuos, patrones de consumo y rangos de edad según la ATSDR (ATSDR, 2003; ATSDR, 2016) (Tabla 3). Se consideró que la biodisponibilidad de fluoruros es total y que el agua potable es la única fuente de ingestión de fluoruros.

Tabla 3. Estimación de la exposición a fluoruros en las distintas comunidades rurales (rangos de edad y peso de acuerdo con Environment Canada, 1993). EEF (mg/kg/día).

\begin{tabular}{|l|c|c|c|c|c|c|}
\hline \multicolumn{1}{|c|}{$\begin{array}{c}\text { Comunidad } \\
\text { pozo }\end{array}$} & $\begin{array}{c}\mathbf{F}^{-} \\
(\mathbf{m g} / \mathbf{L})\end{array}$ & EEF & EEF & EEF & EEF & EEF \\
\hline W(ind) kg) & & 6 & 13 & 27 & 55 & 70 \\
\hline $\mathrm{C}\left(\mathrm{H}_{2} \mathrm{O}\right)(\mathrm{l} / \mathrm{d})$ & & 0.1 & 0.8 & 1.1 & 1.1 & 1.5 \\
\hline CG & 3 & 0.05 & 0.18 & 0.12 & 0.06 & 0.06 \\
\hline Val & 2.36 & 0.04 & 0.15 & 0.1 & 0.05 & 0.05 \\
\hline Gpe & 1.37 & 0.02 & 0.08 & 0.06 & 0.03 & 0.03 \\
\hline DN & 0.94 & 0.02 & 0.06 & 0.04 & 0.02 & 0.02 \\
\hline SJCruz & 0.602 & 0.01 & 0.04 & 0.02 & 0.01 & 0.01 \\
\hline BV & 0.51 & 0.01 & 0.03 & 0.02 & 0.01 & 0.01 \\
\hline
\end{tabular}

Los resultados se emplearon en el análisis de exposición a fluoruros; para esto se consideraron los umbrales de ingesta para los efectos tóxicos de una sustancia cuando un individuo se somete a una exposición crónica por vía oral sugeridos por la ATSDR (ATSDR, 1993; ATSDR, 2003; ATSDR, 2016). Los resultados obtenidos del monitoreo de pozos indican que la 
población ha estado expuesta de manera crónica (más de un año), por lo que los límites de ingesta corresponden a las condiciones de la dosis oral de referencia (RfD), donde el orden de magnitud es un estimado de la exposición diaria que puede tener un individuo a una sustancia tóxica a lo largo de toda su existencia sin presentar daños en ningún órgano, la ingesta máxima de un tóxico que puede tener el ser humano sin presentar manifestaciones clínicas adversas (NOAEL), y la ingesta mínima del agente tóxico que causa manifestaciones clínicas adversas en el individuo expuesto (LOAEL).

El valor que la ATSDR (ATSDR, 1993; ATSDR, 2003) considera para el NOAEL es de $0.15 \mathrm{mg}$ fluoruros/ $\mathrm{kg} /$ día y el valor de LOAEL es de $0.25 \mathrm{mg}$ fluoruros $/ \mathrm{kg} /$ día para observar efectos en el esqueleto (incremento de fracturas); para el caso de NOAEL, se divide por un factor de incertidumbre de tres, para considerar la variabilidad en humanos y así se obtiene un valor de RfD $=0.05$ ( $\mathrm{mg} / \mathrm{kg} /$ día). EPA (IRIS, 2003) derivó una RfD de $0.06 \mathrm{mg} / \mathrm{kg} /$ día para fluoruros basados en un valor de NOAEL de $0.06 \mathrm{mg} / \mathrm{kg} /$ día y de un valor de LOAEL de $0.12 \mathrm{mg} / \mathrm{kg} /$ día para efectos cosméticos de fluorosis dental. El valor de NOAEL fue dividido por un factor de incertidumbre de 1 , a fin de obtener el RfD. Para este trabajo se consideraron los siguientes valores de RfD: $0.05 \mathrm{mg} / \mathrm{kg} /$ día y 0.12 $\mathrm{mg} / \mathrm{kg} /$ día, como valores con los cuales se pueden observar afectaciones. A partir de los valores anteriores, si se obtiene un valor de EEF $>0.05$ ( $\mathrm{mg} / \mathrm{kg} / \mathrm{día})$, los individuos expuestos pueden desarrollar fluorosis dental, dependiendo de la magnitud, dosis y tiempo de exposición. Este valor significa el nivel mínimo de riesgo calculado por la ATSDR (ATSDR, 2003; ATSDR, 2016) para una exposición oral crónica y se considera como el umbral tóxico que ocasiona la aparición de fluorosis dental. En caso de que el valor obtenido sea EEF > 0.12 ( $\mathrm{mg} / \mathrm{kg} /$ día) y el tiempo de exposición sea mucho mayor a 15 años, el individuo presenta un riesgo potencial para desarrollar fluorosis esquelética (ATSDR, 2003; ATSDR, 2016; IRIS, 2003). Este valor significa un nivel de riesgo para desarrollar fluorosis esquelética, considerando la dosis diaria de exposición estimada oral por día.

En Cerro Gordo, Valencia, Dulces Nombres y Santa María Guadalupe, el valor de EEF calculado excede el nivel de $0.05 \mathrm{mg} / \mathrm{kg} /$ día para los distintos rangos de edad (Tabla 3). Ello significa que dichas comunidades están en riesgo potencial de desarrollar fluorosis dental. En Cerro Gordo y Valencia, el valor calculado para EEF excede el nivel de $0.12 \mathrm{mg} / \mathrm{kg} / \mathrm{día}$, principalmente en niños (Tabla 3). Así, los niños están en riesgo potencial de desarrollar fluorosis esquelética. Se debe considerar que una excesiva exposición a fluoruros puede generar diversas afectaciones, donde la de mayor prevalencia es fluorosis dental; de hecho, se ha comprobado que 
el consumo prolongado de agua con contenidos superiores a $1.5 \mathrm{mg} / \mathrm{l}$ de $\mathrm{F}^{-}$es causa de fluorosis dental.

\section{Encuesta epidemiológica sin biomarcadores}

Se analizaron los patrones de consumo relacionados con la ingesta de agua para obtener una visión general del rol del agua potable en la salud de la población, y su riesgo para desarrollar fluorosis dental y esquelética. La mayoría de la población (97.2\%) está conectada al sistema de agua potable local; estos resultados concuerdan con el Anuario Estadístico y Geográfico de Guanajuato (INEGI, 2017). El agua es extraída a través de pozos profundos en cada comunidad rural; dichos pozos están controlados por el Comité Municipal de Agua Potable de JR (CMAPAJ) desde 1995. El $55 \%$ de la población (175 personas) bebe agua directamente del suministro de los pozos, mientras que el $44.8 \%$ (142 personas) bebe agua tratada, o compra garrafones, botellas de agua o refrescos (unos dos litros o menos); estos datos coinciden con el promedio estimado que propone la ATSDR (ATSDR, 2003; ATSDR, 2016).

El agua subterránea es tratada y vendida por compañías locales usando un sistema de potabilización adecuado. La Secretaría de Salud verifica la calidad y el método de tratamiento. Las plantas de tratamiento locales de agua generan residuos con altos contenidos de $\mathrm{F}^{-}$, cuyo destino es desconocido.

El consumo de agua también implica la preparación de alimentos. En este sentido, el $79.2 \%$ (251 personas) usa agua directamente del suministro de los pozos para la cocción de sus alimentos, mientras que $20.80 \%$ (66 personas) usa agua tratada para cocinar (Figura 4a). El 90\% (285 personas) de los individuos percibe un rango de temperatura elevado en el agua que suministran los pozos. Según estudios realizados en el sitio, las altas concentraciones de fluoruros están relacionadas con agua subterránea, que presenta características geotérmicas (Rodríguez et al., 2016; Morales-Arredondo, Rodríguez, Armienta, \& Villanueva-Estrada, 2016a). La temperatura elevada en el agua subterránea varía de 25.5 a $50{ }^{\circ} \mathrm{C}$; los valores más elevados corresponden a pozos localizados cerca de fallas y fracturas; igualmente coinciden estas características con los 
elevados contenidos en $\mathrm{F}^{-}$(Morales-Arredondo, Rodríguez, Armienta, \& Villanueva-Estrada, 2016b).

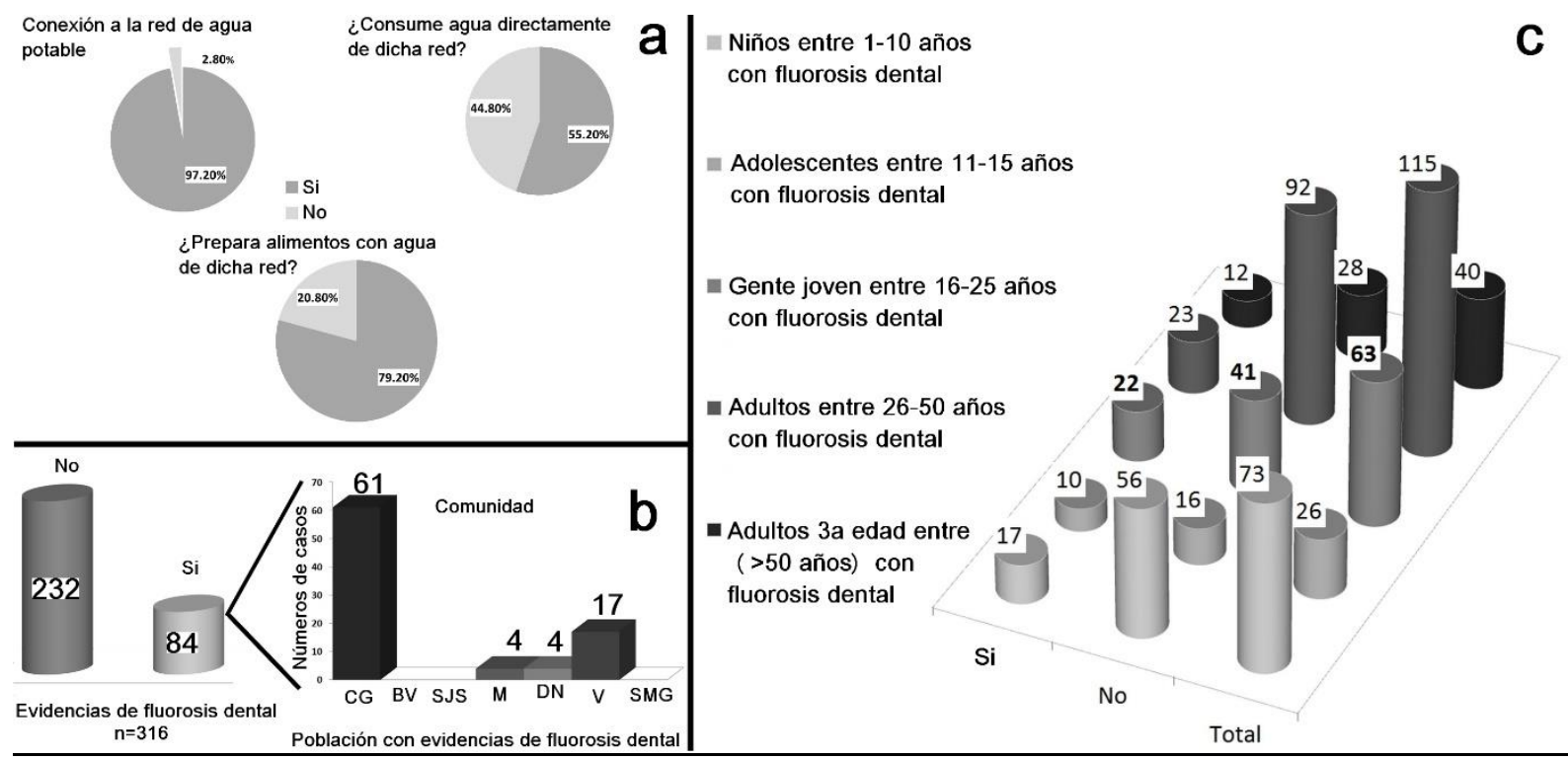

Figura 4. a) Uso del agua a través de la tubería; b) población con evidencias de fluorosis dental en las comunidades rurales CG = Cerro Gordo, BV = Bellavista, SJS = San José del Sauz, M = Murciélago, DN =

Dulces Nombres, V = Valencia, SMG = Santa María Guadalupe; c) población con síntomas relacionados con fluorosis dental por rango de edad.

Doscientas treinta y dos personas $(73.5 \%)$ del total de la población encuestada no manifestaron evidencias de fluorosis dental, pero eso no implica que no estén en riesgo de generar fluorosis, ya que preparan sus alimentos con agua directamente del suministro de los pozos y tienen viviendo en el lugar desde hace más de 10 años; 84 individuos $(26.5 \%)$ presentan evidencias de fluorosis dental, la mayoría de ellos vive en Cerro Gordo ( $72.62 \%, 61$ casos) y Valencia $(20.24 \%, 17$ casos) (Figura 4b). Los pozos en estas comunidades presentan elevados niveles de $\mathrm{F}^{-}$(Figura 1, Figura 2, Figura 3). Además, en estas comunidades, la gente encuestada percibe elevada temperatura en el agua que es suministrada de forma directa de los pozos. Por otro lado, en la zona, la gente consume agua directamente de la llave y prepara alimentos con dicha agua, y/o tienen viviendo más de 10 años en la comunidad. Estas condiciones implican un riesgo para desarrollar fluorosis esquelética (EPA, 2011). Tal información confirma los valores obtenidos por la EEF. 
Algunos autores reportan que niños y personas de la tercera edad pueden ser más vulnerables a desarrollar fluorosis dental (Soto-Rojas et al., 2004; Betancourt-Lineares et al., 2013). Los resultados obtenidos de la EEp muestran que más de $50 \%$ de la población con evidencias de fluorosis dental se encuentra en un amplio rango de edad, desde adolescentes hasta adultos (16 a 50 años); esta situación pudiera estar relacionada con el tiempo de exposición de la población, es decir, el problema puede tener muchos años; se estima que la gente ha bebido agua con elevados contenidos de fluoruros desde hace más de 20 años, momento en el que comenzaron a operar los pozos. Sin embargo, no hay información acerca de los niveles históricos de la concentración de $\mathrm{F}^{-}$en el agua subterránea. Consideramos que los resultados obtenidos están relacionados con los patrones de consumo de agua en cada zona. Otro dato interesante es que el mismo número de personas con afectaciones evidentes se dio en hombres y mujeres.

Otro factor que se debe tomar en cuenta es que la gente utiliza esta agua para regar sus parcelas, plantas y para alimentar a sus animales de granja (cabras, ovejas, cerdos, pollos, vacas). Sería importante revisar los niveles de acumulación de fluoruros en animales y plantas (Kumpulainen \& Koivistonen, 1977; Toth \& Sugar, 1978; Koivistonen, 1980; EPA, 2011), pues de acumular en sus organismos contenidos de $\mathrm{F}^{-}$, podría constituir otra ruta de exposición.

Sería conveniente realizar algún proceso de remoción de fluoruros en el agua de pozos. En el área de estudio se ha reportado la presencia de depósitos de caolinita y material arcilloso, como zeolitas, además de calizas de la Formación Soyatal (CEAG, 2000; INEGI, 2017). Experimentalmente, estos materiales han dado buenos resultados en la disminución de altos contenidos de $\mathrm{F}^{-}$en agua subterránea (Montiel-Cota, Balcázar-Meza, Fernández-Ramírez, Morales-Munguía, \& Varela-Salazar, 2013; Valenzuela, Ramírez-Hernández, Sol, \& Reyes, 2011; CalderónMólgora, Quezada-Jiménez, Segura-Beltrán, \& Hernández-Yañez, 2012; Labastida et al., 2017).

\section{Conclusiones}


Los resultados de la concentración de fluoruros muestran que los habitantes de algunos poblados de Juventino Rosas están expuestos a agua subterránea con elevados contenidos de dicho elemento.

Los resultados de EEF y la EEp sugieren que los habitantes de Cerro Gordo, Valencia, Guadalupe y Dulces Nombres están en riesgo potencial de generar fluorosis dental debido al consumo de agua potable con elevadas concentraciones de $\mathrm{F}^{-}$, pues exceden el mínimo estimado del nivel de riesgo por ingestión propuesto por la ATSDR.

Estos resultados refuerzan los valores obtenidos por la EEp, donde se observaron evidencias de fluorosis dental en la población (un cuarto del total de personas encuestadas), y que puede estar relacionada directamente con el consumo y/o preparación de alimentos con agua que es suministrada directamente de los pozos.

Las evidencias indican que hay incidencia de fluorosis dental en individuos de todas las edades, niños, adultos y gente mayor de 50 años de edad; esto puede deberse al largo tiempo de exposición a los elevados contenidos de $\mathrm{F}^{-}$y puede estar relacionada con el incremento de la extracción de agua subterránea en los años 90, lo que ocasionó el abatimiento de los niveles freáticos en los pozos de toda la zona; esta situación originó una mezcla de agua somera con agua más profunda, la cual presenta mala calidad.

Las comunidades conocen la presencia de $\mathrm{F}^{-}$en el agua potable. La percepción general es que todo el problema se resume a cuestiones estéticas $y$, por lo tanto, la gente continúa bebiendo agua directamente del suministro.

La EEp no fue realizada en las zonas urbanas en este estudio debido a que la comunidad tiene una planta de tratamiento; además, la gente de dicha zona tiene una mejor situación económica, aunque hay que dejar claro que algunos de los pozos urbanos contienen concentraciones de $\mathrm{F}^{-}$ elevadas.

Las campañas de monitoreo y muestreo continúan actualmente y se han detectado altos contenidos de $\mathrm{F}^{-}$en municipios cercanos, como Salamanca - San Miguel de Allende. Sería conveniente que estudios futuros incluyeran también a la población urbana, a fin de tener un más completo panorama del problema.

Una mejor evaluación del impacto debe centrarse en una evaluación más detallada del estado de salud de los pobladores expuestos. 


\section{Agradecimientos}

Agradecemos a Olivia Cruz, Alejandra Aguayo y Nora Ceniceros por su participación en los análisis químicos. Asimismo, al personal del Comité Municipal de Agua Potable de JR (CMAPAJ), que facilitaron el acceso a los pozos. Agradecemos a Flores-Ocampo IZ y Flores-Vargas R. por su participación en las campañas de muestreo.

\section{Referencias}

American Public Health Association, American Water Works Association, Association Water Environment Federation, APHA-AWWA-WWF. (2005). Standard methods for the Examination of Water and Wastewater. Washington, DC, USA: American Public Health Association, American Water Works Association, Association Water Environment Federation.

Agency for Toxic Substances and Disease Registry, ATSDR. (1993). Toxicological profile for fluoride, hydrogen fluoride, and fluorine $(F)$. Atlanta, USA: Department of Health and Human Services, Public Health Service, Agency for Toxic Substances and Disease Registry.

Agency for Toxic Substances and Disease Registry, ATSDR. (2003). Toxicological profile for fluoride, hydrogen fluoride, and fluorine (F). Recovered from https://www.atsdr.cdc.gov/toxprofiles/tp11-c3.pdf

Agency for Toxic Substances and Disease Registry, ATSDR. (2016). Resúmenes de salud pública (fluoruros, fluoruros de hidrógeno, flúor). Recuperado de https://www.atsdr.cdc.gov/es/phs/es_phs11.html

Alarcón-Herrera, M. T., Martín-Domínguez, I. R., Trejo-Vázquez, R., \& Rodríguez-Dozal, S. (2001). Well water fluoride, dental fluorosis, and bone fractures in the Guadiana Valley of Mexico. Fluoride, 34(2), 139149.

Armienta, M. A., \& Segovia, N. (2008). Arsenic and fluoride in the groundwater of Mexico. Environmental Geochemistry and Health, 30, 345353.

Betancourt-Lineares, A., Irigoyen-Camacho, M. E., Mejía-González, A. M., Zepeda-Zepeda, M., \& Sánchez-Pérez, L. (2013). Prevalencia de fluorosis dental en localidades mexicanas ubicadas en 27 estados y el D.F. a seis 
años de la publicación de la Norma Oficial para la fluoruración de la sal. Revista de Investigación Clínica, 65(3), 237-247.

Calderón-Mólgora, C., Quezada-Jiménez, M. L., Segura-Beltrán, N., \& Hernández-Yañez, C. (febrero-marzo, 2012). Remoción de arsénico mediante procesos de membrana. Tecnología y Ciencias del Agua, 3(número especial), 37-51.

Cerca-Martínez, L. M., Aguirre-Díaz, G. J., \& López-Martínez, M. (2000). The geological evolution of the southern Sierra de Guanajuato; a documented example of the transition from the Sierra Madre Occidental to the Mexican Volcanic Belt. International Geology Review, 42, 131-151.

Cervantes-González, M. E., Ortíz-Burgos, J. J., \& Wilbert-Ovalle, J. (1998). Concentración de flúor de ppm de los pozos de agua potable y agua embotelladas de la ciudad de Salamanca, Guanajuato. Revista de la Asociación Dental Mexicana, 55(1), 18-20.

Comisión Estatal del Agua de Guanajuato, CEAG. (2000). Actualización del balance subterráneo de los acuíferos de Guanajuato. Reporte Interno. Guanajuato, México: Comisión Estatal del Agua de Guanajuato.

Díaz-Barriga, F., Navarro-Quezada, A., Grijalva, M. I., Grimaldo, M., Loyola-Rodríguez, J. P., \& Ortíz, M. D. (1997). Endemic fluorosis in México. Fluoride, 30(4), 233-239.

Environment Canada. (1993). Canadian environmental protection act priority substances list assessment report: Arsenic and its compounds. Ottawa, Canada: Canada Communication Group.

Environmental Protection Agency, EPA. (2011). Community water fluoridation, frecuently asked questions. Recovered from www.epa.gov.

Dean, H. T. (1934). Classification of mottled enamel diagnosis. JADA, The Journal of the American Dental Association, 21(8), 1421-1426.

Gómez, J. (1990). Metodología de encuestas por muestreo. En: Arnau, J., Angera, M. T., \& Gómez, J. (eds.). Metodología de la investigación en ciencias del comportamiento (239-309 pp.). Murcia, España: Universidad de Murcia.

Griffin, S. O., Beltran, E. D., Lockwood, S. A., \& Barker, L. K. (2002). Esthetically objectionable fluorosis attributable to water fluoridation. Community Dentistry and Oral Epidemiology, 30, 199-209. 
Grijalva-Haro, M. I., Barba-Leyva, M. E., \& Laborín-Alvarez, A. (2001). Ingestión y excreción de fluoruros en niños de Hermosillo, Sonora, México. Salud Pública de México, 43(2), 127-134.

Grimaldo, M., Borja-Aburto, V. H., Ramírez, A. L., Ponce, M., Rosas, M., \& Díaz-Barriga, F. (1995). Endemic fluorosis in San Luis Potosi, Mexico: I. Identification of risk factors associated with human exposure to fluoride. Environmental Research, 68, 25-30.

Hurtado-Jiménez, R., \& Gardea-Torredey, J. (2005). Estimación de la exposición a fluoruros en Los Altos Jalisco, México. Salud Pública de México, 47(1), 58-63.

Instituto Nacional de Estadística y Geografía, INEGI. (2017). Anuario Estadístico y Geográfico de Guanajuato 2016. Guanajuato, México: Instituto Nacional de Estadística y Geografía, Gobierno del Estado de Guanajuato.

Integrated Risk Information System, IRIS. (2003). Fluorine (soluble fluoride). Washington, DC, USA: Integrated Risk Information System, U.S. Environmental Protection Agency.

Kish, L. (1982). Muestreo de encuestas (3a reimp.). México, DF, México: Editorial Trillas.

Koivistonen, P. (1980). Mineral element composition of Finnish foods. Acta Agriculturae Scandinavica, 22, 1-171.

Kumpulainen, J., \& Koivistonen, P. (1977). Fluorine in foods. Residue Reviews, 68, 37-57.

Labastida, I., Armienta, M. A., Beltrán, M., Caballero, G., Romero, P., \& Rosales, M. A. (2017). Limestone as a sustainable remediation option for water contaminated with fluoride. Journal of Geochemical Exploration. Recuperado http://dx.doi.org/10.1016/j.gexplo.2016.12.001, 2017

Levy, D. B., Schramke, J. A., Esposito, K. J., Erickson, T. A., \& More, J. C. (1999). The shallow groundwater chemistry of arsenic, fluorine, and major elements: Eastern Owens Lake, California. Applied Geochemistry, $14,53-65$.

McDonagh, M. S., Whiting, P. F., Wilson, P. M., Sutton, A. J., Chestnutt, I., Cooper, J., Misso, K., Bradley, M., Treasure, E., \& Kleijinen, J. (2000). Systematic review of water fluoridation (CRD Report 18). Heslington, UK: NHS Centre for Reviews and Dissemination. 
Mendoza, E. (1999). Diagnóstico de la calidad del agua potable en las zonas urbanas del estado de Guanajuato (tesis de Maestría en Protección y Conservación Ambiental). Universidad Iberoamericana, León, Guanajuato, México.

Montiel-Cota, A., Balcázar-Meza, M., Fernández-Ramírez, M. V., Morales-Munguía, J. C., \& Varela-Salazar, J. (2013). Eliminación de flúor en agua potable utilizando zeolita natural (Clinoptilolita). Biotecnia, $15(2), 10-14$.

Morales, I., Villanueva-Estrada, R. E., Rodríguez, R., \& Armienta, M. A. (2015). Geological, hydrogeological, and geothermal factors associated to the origin of arsenic, fluoride, and groundwater temperature in a volcanic environment "El Bajío Guanajuatense" Mexico. Environmental Earth Sciences, 74, 5403-5415.

Morales-Arredondo, I., Rodríguez, R., Armienta, M. A, \& VillanuevaEstrada, R. E. (2016a). A low-temperature geothermal system in central Mexico: Hydrogeochemistry and potential heat source. Geochemical Journal, 50(3), 211-225.

Morales-Arredondo, I., Rodríguez, R., Armienta, M. A., \& VillanuevaEstrada, R. E. (2016b). The origin of groundwater arsenic and fluorine in a volcanic sedimentary basin in central Mexico: a Hydrochemistry hypothesis. Hydrogeology Journal, 25(1), 1-16.

Méndez-Ramírez, I., Namihira, G. D., Moreno, L., \& Sosa-de-Martinez, C. (2001). El protocolo de investigación: lineamientos para su elaboración y análisis. México, DF, México: Editorial Trillas.

NOM-127-SSA1-1994: Norma Oficial Mexicana "Salud ambiental, agua para uso y consumo humano-límites permisibles de calidad y tratamientos a que debe someterse el aguapara su potabilización" (Modificada 2000). $1-7$.

NOM-230-SSA1-2002: Norma Oficial Mexicana. "Salud ambiental. Agua para uso y consumo humano, requisitos sanitarios que se deben cumplir en los sistemas de abastecimiento públicos y privados durante el manejo del agua. Procedimientos sanitarios para el muestreo". Recuperada de http://www.salud.gob.mx/unidades/cdi/nom/230ssa102.html

Orozco-Esquivel, M. T., Nieto-Samaniego, A. F., \& Alaniz-Alvarez, S. A. (2002). Origin of rhyolitic lavas in the Mesa Central, Mexico, by crustal melting related to extension. Journal of Volcanology and Geothermal Research, 118, 37-56. 
Ortíz-Burgos, M., Vargas-Garcidueñas, D., \& Ovalle-Castro, J. (nov.-dic., 1996). Fluorosis dental de la población escolar de Salamanca Guanajuato. Revista ADM, Órgano Oficial de la Asociación Dental Mexicana, 53(6), 289294.

Ortíz, D., Castro, L., Turrubiates, F., Milan, J., \& Díaz-Barriga, F. (1998). Assessment of the exposure to fluoride in drinking water from Durango, Mexico, using a Geographic Information System. Fluoride, 31(4), 183187.

Petersen, E., Essene, D., \& Peacor, D. (1982). Fluorine end-member micas and amphiboles. American Mineralogist, 67, 538-544.

Quintero, O. (1999). Evolución geológica del extremo noroccidental de la Sierra de Guanajuato. Instituto de Geología, UNAM. Resúmenes y Guía de Excursiones. Simposio sobre la Geología de la Sierra de Guanajuato, Guanajuato, México.

Ravishankar, S. (1987). Status of geothermal exploration in Maharashtra and Madhya Pradesh (C.R.). GSI Rec, 115(6), 7-29.

Rivett, M., Drewes, J., Barrett, M., Chilton, J., Appleyard, S., Dieter, H. H., Wauchope, D., \& Fastner, J. (2006). Chemicals: Health relevance, transport and attenuation, in protecting groundwater for health managing the quality of drinking-water source. In: Schmon, O., Howard, G., Chilton, J., Chorus, I., \& World Health Organization (eds.). Protecting groundwater for health: Managing the quality of drinking-water sources. Recuperado de http://www.who.int/iris/handle/10665/43186

Rodríguez, R., Reyes, R., Rosales, J., Berlín, J., Mejía, J. A., \& Ramos, A. (2001). Estructuración de mapas temáticos de índices de vulnerabilidad acuífera de la mancha urbana de Salamanca Gto. Municipio de Salamanca, reporte técnico. México, DF, México: Comisión Estatal del Agua de Guanajuato, Instituto de Geofísica-Universidad Nacional Autónoma de México.

Rodríguez, R., Morales, I., Armienta, A., Villanueva, R., \& Segovia, N. (2015). Geothermal systems of low temperature in Mexican Highlands: Alternative uses and associated risks. Procedia Environmental Sciences, 25, 214-219.

Rodríguez, R., Morales-Arredondo, I., \& Rodríguez, I. (2016). Geological differentiation of groundwater threshold concentrations of arsenic, vanadium and fluorine in El Bajío Guanajuatense, México. Geofísica Internacional, 55, 5-15. 
Rubio, P. (2009). Metodología y aplicabilidad de las encuestas seroepidemiológicas; agrupación de enfermedades infecciosas y salud internacional del CIBER en epidemiología y salud pública (CIBERESP) (Taller 2009, Barcelona). Revista Especializada en Salud Pública, 83(5), 611-623.

Ruiz, J., Kesler, S., \& Jones, L. (1985). Strontium isotope geochemistry of fluorite mineralization associated with fluorine-rich igneous rocks from The Sierra Occidental, Mexico; possible exploration significance. Economic Geology, 80, 33-42.

Sistema de Agua Potable y Alcantarillado de San Miguel Allende, SAPASMA. (2006). Well water quality in San Miguel de Allende Phase I: Results and conclusions. Recuperado de http://www.ecosystemsciences.com/Portals/0/pdfs/Drinking\%20Water \%20Quality\%20in\%20San\%20Miguel,\%20Mexico.pdf

Soto-Rojas, A. E., Ureña-Cirret, J. L., \& Martinez-Mier, E. A. (2004). A review of the prevalence of dental fluorosis in Mexico. Revista Panamericana de Salud Pública, 15, 9-17.

Toth, K., \& Sugar, E. (1978). Fluorine content of foods and the estimated daily intake from foods. Acta Physiologica, 51, 361-369.

Trejo-Vázquez, R., \& Bonilla-Petriciolet, A. (2001). Exposición a fluoruros del agua potable en la ciudad de Aguascalientes, México. Pan American Journal of Public Health, 10(2), 108-113.

Valenzuela, L., Ramírez-Hernández, J., Sol, J. A., \& Reyes, J. A. (2011). Alternativas para la eliminación doméstica de flúor en el agua de consumo humano. Información Tecnológica, 22(2), 23-32. 